\title{
Sylvestre, Paul-François. Cinquante ans de «p'tits bonheurs » au Théâtre français de
}

\section{Toronto. Toronto : Gref, 2016. 279 p.}

En 1967, le Canada célèbre le centième anniversaire de la confédération. Un large programme de festivités est prévu à travers le pays, entre autres l'Expo 67 à Montréal. Mais Toronto n'est pas en reste et c'est dans cette ambiance qu'est fondé le Théâtre du p'tit bonheur qui devient en 1987 le Théâtre français de Toronto. Cinquante ans de "p'tits bonheurs » au Théâtre français de Toronto retrace les histoires, succès et émotions de cette troupe professionnelle caractérisée par son dynamisme au service d'un public minoritaire en milieu anglophone.

L'ouvrage forme une archive riche en photographies (couleurs, noir et blanc) qui documente les activités croissantes d'un projet initié par la Fédération des femmes canadiennes françaises (FFCF). Les amoureux de théâtre et les amateurs d'histoire locale se régaleront à consulter la programmation complète de 1967 à 2017 (pour chaque pièce sont inclus les dates, la mise en scène, la distribution et, autant que possible, le résumé), ainsi que divers témoignages d'individus qui ont contribué à l'épanouissement de la compagnie (entre autres, la co-fondatrice Claudette Roy-Gobeil, le comédien Raymond Accolas, le scénographe Glen Charles Landry, la coach d'écriture Patricia Marceau, le régisseur Gabriel Dubé, la costumière Nina Okens). Cinquante ans de "p'tits bonheurs » au Théâtre français de Toronto note la prédilection de la troupe pour Molière et Michel Tremblay, mais établit aussi la longue liste de dramaturges joués au cours des saisons, depuis Alexis Arbuzov à Anton Tchekhov, en passant par Yasmina Reza, Martin Bellemare ou encore Marie Laberge.

Rien n'échappe à l'auteur et les lecteurs pourront prendre connaissance, non seulement de la liste des honneurs et distinctions attribués à la troupe, mais aussi du profil des directeurs artistiques et de la composition des conseils d'administration, auxquels s'ajoutent la (longue) liste des élèves ontariens dont les textes ont été joués dans la série des Zinspirés, les revenus et dépenses d'une décennie à l'autre et des analyses sur la presse francophone et anglophone.

Cet ouvrage de Paul-François Sylvestre est à la fois portrait et panorama, historique et catalogue et, en fin de compte, passion vérifiée par les faits.

Emilienne Rue 